\title{
The pituitary adenylate cyclase-activating polypeptide is a physiological inhibitor of platelet activation
}

\author{
Kathleen Freson, ${ }^{1}$ Hitoshi Hashimoto, ${ }^{2}$ Chantal Thys, ${ }^{1}$ Christine Wittevrongel, ${ }^{1}$ Sophie Danloy, ${ }^{1}$ \\ Yoshiko Morita, ${ }^{2}$ Norihito Shintani, ${ }^{2}$ Yoshiaki Tomiyama, ${ }^{3}$ Jos Vermylen, ${ }^{1}$ Marc F. Hoylaerts, ${ }^{1}$ \\ Akemichi Baba, ${ }^{2,4}$ and Chris Van Geet ${ }^{1,5}$

\begin{abstract}
${ }^{1}$ Center for Molecular and Vascular Biology, University Hospital Gasthuisberg, University of Leuven, Leuven, Belgium. ${ }^{2}$ Laboratory of Molecular Neuropharmacology, Graduate School of Pharmaceutical Sciences, ${ }^{3}$ Department of Internal Medicine and Molecular Science, Graduate School of Medicine, and 4Laboratory of Molecular Pharmacology, Graduate School of Medicine, Osaka University, Suita, Osaka, Japan. ${ }^{5}$ Department of Pediatrics, University Hospital Gasthuisberg, University of Leuven, Leuven, Belgium.
\end{abstract}

\begin{abstract}
The pituitary adenylate cyclase-activating polypeptide (PACAP) is a neuropeptide of the vasoactive intestinal peptide/secretin/glucagon superfamily. Studies in two related patients with a partial trisomy 18p revealed three copies of the PACAP gene and elevated PACAP concentrations in plasma. The patients suffer from severe mental retardation and have a bleeding tendency with mild thrombocytopenia, and their fibroblasts show increased PACAP mRNA levels. The PACAP receptor (vasoactive intestinal peptide/pituitary adenylate cyclase-activating peptide receptor 1 [VPAC1]) in platelets and fibroblasts is coupled to adenylyl cyclase activation. Accordingly, we found increased basal cAMP levels in patients' platelets and fibroblasts, providing a basis for the reduced platelet aggregation in these patients. Megakaryocyte-specific transgenic overexpression of PACAP in mice correspondingly increased PACAP release from platelets, reduced platelet activation, and prolonged the tail bleeding time. In contrast, the PACAP antagonist PACAP(6-38) or a monoclonal PACAP antibody enhanced the collagen-induced aggregation of normal human platelets, and in PACAP knockout mice, an increased platelet sensitivity toward collagen was found. Thus, we found that PACAP modulates platelet function and demonstrated what we believe to be the first hemostatic defect associated with PACAP overexpression; our study suggests the therapeutic potential to manage arterial thrombosis or bleeding by administration of PACAP mimetics or inhibitors, respectively.
\end{abstract}

\section{Introduction}

The pituitary adenylate cyclase-activating polypeptide (PACAP) was first isolated from ovine hypothalamic extracts on the basis of its ability to stimulate CAMP formation in rat anterior pituitary cells $(1,2)$. This neuropeptide bears partial sequence homology with vasoactive intestinal peptide (VIP), growth hormone-releasing hormone, secretin, and glucagon. Its role in biology seems crucial, since the sequence of PACAP is highly conserved during the evolution from protochordates to mammals. Like other hypophysiotropic neurohormones, PACAP is contained in extrahypothalamic neurons as well as in numerous peripheral tissues. Consistent with its widespread distribution, PACAP has been found to exert pleiotropic effects including modulation of neurotransmitter release, vasodilation, bronchodilation, activation of intestinal motility, increase of insulin and histamine secretion, and stimulation of cell multiplication and/or differentiation (2). PACAP therefore is believed to act as a hormone, a neurohormone, a neurotransmitter, and a trophic factor in different tissues.

The human PACAP gene is located on chromosome 18p11.32 and encodes a 176-amino acid prepro-protein, which comprises a

Nonstandard abbreviations used: acid citrate dextrose (ACD); adenylyl cyclase-stimulatory G protein (Gs); Friend leukemia virus, strain B (FVB); 3-isobutyl 1-methylxanthine (IBMX); pituitary adenylate cyclase-activating polypeptide (PACAP); platelet-poor plasma (PPP); platelet-rich plasma (PRP); regulator of G protein signaling-2 (RGS2); vasoactive intestinal peptide (VIP); VIP/pituitary adenylate cyclase-activating peptide receptor 1 (VPAC1).

Conflict of interest: The authors have declared that no conflict of interest exists. Citation for this article: J. Clin. Invest. 113:905-912 (2004). doi:10.1172/JCI200419252. 24-amino acid signal peptide (3). In all mammalian species studied so far, the sequences of the active PACAP peptides PACAP(1-38) and its derived form PACAP(1-27) are located in the C-terminal domain of the precursor. Both forms were found to be equipotent in receptor-binding and adenylyl cyclase assays (4). The active peptide PACAP(1-38) is mainly expressed in testis and brain, but this peptide can cross the blood-brain barrier and is stably transported in plasma through coupling to ceruloplasmin (5-7).

PACAP(1-38) and PACAP(1-27) are ligands for three $G$ protein-coupled receptors: the PACAP-specific PAC1 receptor, which is coupled to several transduction systems and mainly expressed in the CNS, and the PACAP/VIP-indifferent VIP/pituitary adenylate cyclase activating peptide receptor 1 (VPAC1) and VPAC2 receptors, which are primarily coupled to adenylyl cyclase and widely distributed in peripheral tissues (8-11). The wide distribution of PACAP receptors has led to an explosion of studies aimed at determining the pharmacological effects and biological functions of this peptide. PACAP has presently been investigated in many organs and tissues, such as endocrine glands, CNS, respiratory system, cardiovascular system, and gastrointestinal tract (2). Extensive studies have also been performed on its function in the immune system, but no data are currently available to support a function for PACAP in hemostasis. Only one study reports that PACAP inhibits the cyclooxygenase pathway of rat platelets via a specific PACAP receptor-activated, cAMP-dependent pathway (12).

We previously have described several unrelated patients with a novel genetic defect and inducible hyperactivity of the adenylyl cyclase-stimulatory $G$ protein $(G s)(13,14)$. These patients have an increased Ivy 
bleeding time and other abnormalities, including a variable degree of mental retardation and mild skeletal malformations. Modest stimulation via different Gs-coupled platelet receptors in these patients triggers strong and rapid adenylyl cyclase activation, thereby antagonizing platelet activation. Because we found that hyperfunction of the Gs $\alpha$ pathway could lead to a bleeding phenotype (13), we have investigated how a partial trisomy $18 \mathrm{p}$, which results in overexpression of the Gs agonist PACAP and is also associated with a pronounced bleeding phenotype, affects platelet function. We found that PACAP is a natural regulator of platelet activation. This rather unexpected role of PACAP in hemostasis was further confirmed in both PACAP knockout mice and mice with megakaryocyte-specific overexpression of PACAP.

\section{Methods}

Generation and genotyping of mice with megakaryocyte-specific overexpression of PACAP and Southern blot analysis. The murine GPIIb promoter (extending from +23 to 508 relative to the initiation start site) was excised from the mGPIIb-pGL3 plasmid by digestion with KpnI and BamHI and inserted into the KpnI-BamHI-digested PACAPpcDNA3.1 vector (Invitrogen Corp., Carlsbad, California, USA) in front of the mouse PACAP gene (15). The mouse PACAP gene was amplified from brain CDNA with primers mPACAP1R 5'-GTAGCCGCTCGAGGATCTGCTACAAGTATGC- $3^{\prime}$ and mPACAP4F $5^{\prime}$ GTTAGCCGAATTCAGTTCAAGGTCTGGCTAG-3', sequenced, and cloned into the EcoRI-XhoI site of the pcDNA3.1 vector. The $2.2-\mathrm{kb}$ KpnI-DraIII fragment (GPIIb-PACAP) was excised and purified for zygote injection. The GPIIb promoter has been successfully used to restrict transgene expression to the megakaryocytic cell lineage of mice (16). Transgenic PACAP-overexpressing mice were generated by zygote injection into the Friend leukemia virus, strain B (FVB) background according to previously published procedures $(17,18)$. Transgenic offspring were identified by PCR screening using genomic DNA extracted from tail samples. The following primer pair was used: mGPIIb1F 5'-TGGCCACATCACAGCATTCAAG-3' ${ }^{\prime}$ and mPACAP1R. Southern blot analysis was performed to identify founders; $40 \mu \mathrm{g}$ of genomic DNA was digested with SacI (Fermentas GmbH, St. LeonRot, Germany), and PACAP cDNA was used as a probe.

Production of polyclonal and monoclonal antibodies. Primary anti-PACAP $\mathrm{mAb}$ (PP1A4) and anti-PACAP, anti-regulator of $\mathrm{G}$ protein signaling-2 (anti-RGS2), and anti-VPAC1 polyclonal antibodies were produced in our laboratory by injection of mice and rabbits, respectively, with recombinant fusion proteins consisting of the PACAP $(1-38)$ peptide sequence, the complete VPAC1 sequence, or the N-terminal part of the RGS2 sequence, each coupled to glutathione-S-transferase. These recombinant fusion proteins were expressed in Escherichia coli and purified by affinity chromatography on immobilized glutathione (Amersham Biosciences AB, Uppsala, Sweden). The primary antibodies were purified on protein A-Sepharose beads (Amersham Biosciences AB) and controlled for their reactivity toward recombinant PACAP(1-38), VPAC1, or RGS2 by immunoblot.

Human platelet aggregation. Blood was anticoagulated with $3.8 \%$ (wt/vol) trisodium citrate (9:1), and platelet-rich plasma (PRP), obtained by centrifugation ( $150 \mathrm{~g}$ for 15 minutes), was recentrifuged $(3,000 \mathrm{~g}$ for 15 minutes) to produce platelet-poor plasma (PPPtrisodium citrate). Finally, the platelet count in the PRP was adjusted to $250 \times 10^{9}$ platelets per liter with PPP. Platelet aggregation was performed on two dual-channel Chrono-Log aggregometers (Chrono-Log Corp., Havertown, Pennsylvania, USA) by simultaneous recording of four tracings. Aggregation-inhibition studies were done as described previously $(13,14)$ and involved dose-response curves to the stable prostacyclin analogue and Gs agonist iloprost (Ilomédine; 0-5 ng/ml; Schering AG, Berlin, Germany), which was added to PRP 1 minute before induction of aggregation by Horm collagen $(2 \mu \mathrm{g} / \mathrm{ml}$; Nycomed Arzneimittel, Munich, Germany). The $\mathrm{IC}_{50}$ value was calculated after curve fitting using the software InStat 2.03 (GraphPad Software Inc., San Diego, California, USA). The $\mathrm{IC}_{50}$ values \pm SD were compared with those for the same Gs agonist on control platelets, studied simultaneously.

In vivo effect of anti-PACAP antibodies on platelet aggregation in mice. FVB mice were injected three times subcutaneously with $200 \mu \mathrm{g}$ of an anti-PACAP, an anti-RGS2, or an anti-VPAC1 polyclonal antibody or four times with $50 \mu \mathrm{g}$ of an anti-PACAP mAb (PP1A4) with an interval of 3 days between injections. At day 14 after the first injection, whole blood from the inferior vena cava of mice anesthetized by intraperitoneal injection of $60 \mathrm{mg} / \mathrm{kg}$ sodium pentobarbital was drawn into $20 \mu \mathrm{g} / \mathrm{ml}$ hirudin. A platelet count was performed on the venous blood sample using the CELL-DYN 1300 (Abbott Laboratories, Abbott Park, Illinois, USA). Blood was centrifuged at $100 \mathrm{~g}$ for 10 minutes, allowing separation of PRP, and $P$ was obtained by centrifugation of the remaining blood at $2,000 \mathrm{~g}$ for 10 minutes. PRP and $P$ were pooled from five mice in each group. Platelet aggregation was measured as described above with the platelet count adjusted to $250 \times 10^{9}$ platelets per liter. All animal experiments were approved by the institutional review board and were conducted according to $\mathrm{NIH}$ guidelines for animal experiments.

Bleeding time and platelet aggregation in PACAP-overexpressing mice. The bleeding time was determined as described previously (19). Eight- to 15 -week-old mice were bled under sodium pentobarbital anesthesia from the retroorbital plexus. Mouse blood was collected in a saline solution containing $20 \mu \mathrm{g} / \mathrm{ml}$ hirudin. Preparation of PRP and aggregation were done as described above.

Immunoblot detection of PACAP in washed platelet releasates. Blood was collected into trisodium citrate and centrifuged at $200 \mathrm{~g}$ for $15 \mathrm{~min}$ utes at room temperature to yield PRP. PRP from five mice was pooled. Then, $0.1 \mathrm{vol}$ of acid citrate dextrose (ACD; $2.5 \%$ trisodium citrate, $1.5 \%$ citric acid, $2 \%$ D-glucose) was added to the PRP, and platelets were purified from PRP by centrifugation at $700 \mathrm{~g}$ for 15 minutes at room temperature. The platelet pellet was washed and resuspended in $250 \mu \mathrm{l}$ HEPES/Tyrode buffer $(10 \mathrm{mM}$ HEPES, $137 \mathrm{mM} \mathrm{NaCl}, 2.68 \mathrm{mM} \mathrm{KCl}, 0.42 \mathrm{mM} \mathrm{NaH}_{2} \mathrm{PO}_{4}, 1.7 \mathrm{mM} \mathrm{MgCl}_{2}$, $11.9 \mathrm{mM} \mathrm{NaHCO}_{3}, \mathrm{pH} 7.4$ ) containing $5 \mathrm{mM}$ D-glucose at $100 \times 10^{10}$ platelets per liter. Platelets were activated with $10 \mu \mathrm{g} / \mathrm{ml}$ Horm collagen and pelleted. The washed platelet-releasate proteins were resolved by SDS-PAGE and transferred to Hybond ECL-nitrocellulose membranes (Amersham Biosciences $\mathrm{AB}$ ). Blots were revealed with a monoclonal antiserum against PACAP (PP1A4) and stained with Western blotting ECL detection reagent (Amersham Biosciences AB).

Platelet aggregation in PACAP knockout mice. Generation of PACAP knockout mice has been previously described (20). Blood from the heart of mice anesthetized by intraperitoneal injection of $60 \mathrm{mg} / \mathrm{kg}$ sodium pentobarbital was drawn into $0.1 \mathrm{M}$ citrate, and PRP and $P$ were pooled from three mice. Platelet aggregation was performed on an MCM Hema Tracer 313M model PAM-12C (MC Medical Inc., Tokyo, Japan) using Horm collagen at indicated concentrations.

cAMP detection in human and mouse platelets. We incubated citrated PRP with iloprost $(1 \mathrm{ng} / \mathrm{ml})$, arrested the reaction at different time points by adding $12 \%$ trichloroacetic acid, and measured human platelet cAMP using a cAMP enzyme immunoassay (Amersham Biosciences $\mathrm{AB}$ ). Basal cAMP levels in human platelets were measured in the presence of the phosphodiesterase inhibitor 3-isobutyl 


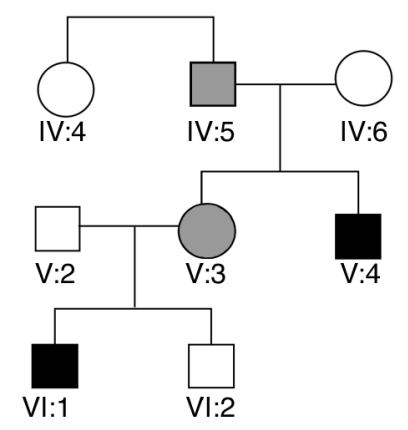

Figure 1

Family presentation. Squares, male; circles, female; filled symbols, affected individuals; open symbols, unaffected individuals. Black filled symbols represent members with severe mental retardation and a partial trisomy $18 p$ and monosomy 20p, and striped symbols represent members with borderline IQ and the balanced translocation $t(18 ; 20)(p 21 ; p 13)$.

1-methylxanthine (IBMX; $100 \mu \mathrm{M}$ final concentration). The accumulation of cAMP in mouse platelets was measured in the absence of IBMX, using a $\left.{ }^{125} \mathrm{I}\right]$ cAMP RIA kit (Amersham Biosciences AB) as previously described (21).

Human skin fibroblasts and cAMP detection. Skin fibroblasts were obtained via punch biopsy from the volar side of the upper arm. Fibroblasts were cultured in DMEM/H12 (Invitrogen Corp.) supplemented with $10 \% \mathrm{FBS}$ and antibiotics (Invitrogen Corp.), at $37^{\circ} \mathrm{C}$ in a $5 \% \mathrm{CO}_{2}$ humidified incubator. Only fibroblasts of low passage number (between 6 and 12) were used for DNA and RNA extraction and for CAMP measurements. Cells were grown to $100 \%$ confluence and then incubated in serum-free medium for 24 hours before cAMP analysis. Fibroblasts were plated in duplicate, one plate being used for the assay, the other for cell counting; the obtained cAMP levels were thus adjusted for the cell number. Patient or control fibroblasts were stimulated with the Gs agonist isoproterenol (CalbiochemNovabiochem Corp., San Diego, California, USA) at $1 \mu \mathrm{M}$ in the presence of a phosphodiesterase inhibitor (IBMX; $100 \mu \mathrm{M})$, and reactions were terminated by the cell lysis buffer supplied with the kit. The cAMP levels were measured using the cAMP enzyme immunoassay mentioned above.

FISH analysis. FISH analysis was performed on metaphase spreads according to standard procedures (22), using a biotinlabeled YAC 841C3 probe (23) including the PACAP gene (ADCYAP1) locus at 18p11.31-32 in combination with two centromeric probes for chromosomes 18 and 20.

Semiquantitative detection of PACAP(1-38) $m R N A$. Total RNA was extracted from cultured fibroblasts using TRIzol reagent according to the manufacturer's protocol (Invitrogen Corp.). Approximately 1 $\mu \mathrm{g}$ of DNase I-treated fibroblast RNA, in the presence of an RNase I inhibitor, was used for oligo-dT-primed first-strand cDNA synthesis using Moloney murine leukemia virus (M-MLV) reverse transcriptase (Invitrogen Corp.). The reverse transcriptase reaction was terminated by heating for 5 minutes at $95^{\circ} \mathrm{C}$. The cDNA content was normalized using primers for $\beta$-actin. The following primer sets were used to generate specific fragments: $\beta$-actin $\beta 5 \mathrm{~F}$ (ACCAACTGGGACGACATGGAG) and $\beta 3$ R (CGTGAGGATCTTCATGAGGTAGTC) and PACAP(1-38) PACAP4F (GAAGCACCTGCAGTCGCTCG) and PACAP2R (TGTATACACAGGGTAGC). All PCR reactions (with 17 and 20 cycles) were also performed in duplicate on separate fibroblast RNA samples.
Detection of PACAP in plasma by ELISA. Blood was anticoagulated with $3.8 \%$ (wt/vol) trisodium citrate (9:1) or ACD, pH 6.5 (9:1), and plasma (PPP) was obtained by centrifugation for 15 minutes at 3,000 g. A polyclonal anti-PACAP antibody was coated overnight at $4{ }^{\circ} \mathrm{C}$ in microtiter plates (Corning-Costar Corp., Cambridge, Massachusetts, USA) at $10 \mu \mathrm{g} / \mathrm{ml}$ in $200 \mu \mathrm{l} \mathrm{PBS}$. After blocking of the plates with $1 \%$ nonfat dry milk in PBS, plasma samples $(0-0.01 \mu \mathrm{l} / 200 \mu \mathrm{l})$ were deposited in the wells overnight at $4^{\circ} \mathrm{C}$ in PBS supplemented with $1 \%$ nonfat dry milk and $0.002 \%$ (vol/vol) Tween-80. Bound PACAP(1-38) was revealed with secondary HRP-conjugated polyclonal anti-PACAP antibody (dilution 1:2,500) and $O$-phenylenediamine. A dilution series of recombinant PACAP(1-38) served as a quantitative standard.

\section{Results}

Patient descriptions. We describe a family characterized by an unbalanced segregation of the reciprocal translocation $\mathrm{t}(18 ; 20)$ (p21;p13), in which several members manifest severe mental retardation (Figure 1). The propositus (VI:1) is a 23-year-old man with multiple neurological problems (epilepsy, hypotonia, convulsions, mental retardation, tremor, psychotic behavior, hyperactive behavior), gastrointestinal problems (diarrhea, vomiting), and endocrinological problems (hypoplasia of the pituitary gland, hypogonadotropic hypogonadism). In addition, he presented on different occasions with a moderate thrombocytopenia, the platelet count being $70 \times 10^{9}$ to $90 \times 10^{9}$ platelets per liter. He has a severe bleeding problem with repetitive epistaxis, even leading to hypovolemic shock, and a markedly prolonged Ivy bleeding time (>15 minutes), though coagulation studies are normal. He has a decreased platelet secretion of ATP and $\beta$-thromboglobulin despite platelet electron microscopy showing a normal amount of dense and $\alpha$ granules. His karyotype shows a partial trisomy 18p and monosomy 20p.

His brother (VI:2), father (V:2), and maternal grandmother (IV:6) are phenotypically normal, have no bleeding problems, and have a normal karyotype. In contrast, his mother (V:3) and maternal grandfather (IV:5) have no obvious neurological abnormalities but borderline IQs. They carry the balanced translocation $t(18 ; 20)$ ( 21 ; 13 13). They have no obvious bleeding problem and have a normal platelet count. The propositus's 47-year-old uncle (V:4) also suffers from severe mental retardation, hypogonadism, pronounced recurrent epistaxis, and cryptorchidism. Furthermore, he frequently has gastric bleeding, and his platelet count is around $150 \times 10^{9}$ platelets per liter. He also has a partial trisomy $18 \mathrm{p}$ and

\section{Table 1}

Platelet aggregation

\begin{tabular}{lcc}
\hline & $\begin{array}{c}\mathbf{I C}_{50} \pm \mathbf{S D}^{\mathrm{A}} \\
\text { Iloprost }(\mathbf{n g} / \mathbf{m l})\end{array}$ & $\begin{array}{c}\mathbf{E C}_{50} \pm \mathbf{S D}^{\mathbf{B}} \\
\text { Collagen }(\mu \mathbf{g} / \mathbf{m l})\end{array}$ \\
$\mathrm{IV}: 6$ & $0.96 \pm 0.002$ & $0.25 \pm 0.012$ \\
$\mathrm{IV}: 5$ & $0.40 \pm 0.003^{\mathrm{C}}$ & $0.74 \pm 0.013^{\mathrm{C}}$ \\
$\mathrm{V}: 3$ & $0.47 \pm 0.003^{\mathrm{C}}$ & $0.75 \pm 0.006^{\mathrm{C}}$ \\
$\mathrm{V}: 4$ & $0.27 \pm 0.005^{\mathrm{D}}$ & $1.08 \pm 0.006^{\mathrm{D}}$ \\
Vl:1 & $0.34 \pm 0.004^{\mathrm{D}}$ & $1.03 \pm 0.008^{\mathrm{D}}$ \\
Controls & $1.04 \pm 0.39(n=22)$ & $0.22 \pm 0.6(n=10)$
\end{tabular}

${ }^{A} C_{50} \pm S D$ values for indicated individuals, and the mean $\mathrm{IC}_{50}$ value for iloprost in the platelet aggregation inhibition test with $2 \mu \mathrm{g} / \mathrm{ml}$ collagen in 22 controls, were calculated. A significantly lower $\mathrm{IC}_{50}$ value $\left({ }^{C} P \leq 0.03\right.$ or $\mathrm{D} P \leq 0.0076)$ indicates Gs hyperfunction. ${ }^{\mathrm{B} T h e}$ right column illustrates the decreased response to collagen of IV:5 and V:3 $\left({ }^{C} P \leq 0.003\right)$ and of V:4 and $\mathrm{VI}: 1$ ( $\mathrm{DP} \leq 0.0001$ ) compared with ten controls. Effector concentration ${ }_{50}$ $\left(\mathrm{EC}_{50}\right)$ is expressed as the collagen concentration that induces aggregation with an amplitude that is $50 \%$ that of maximal aggregation. 

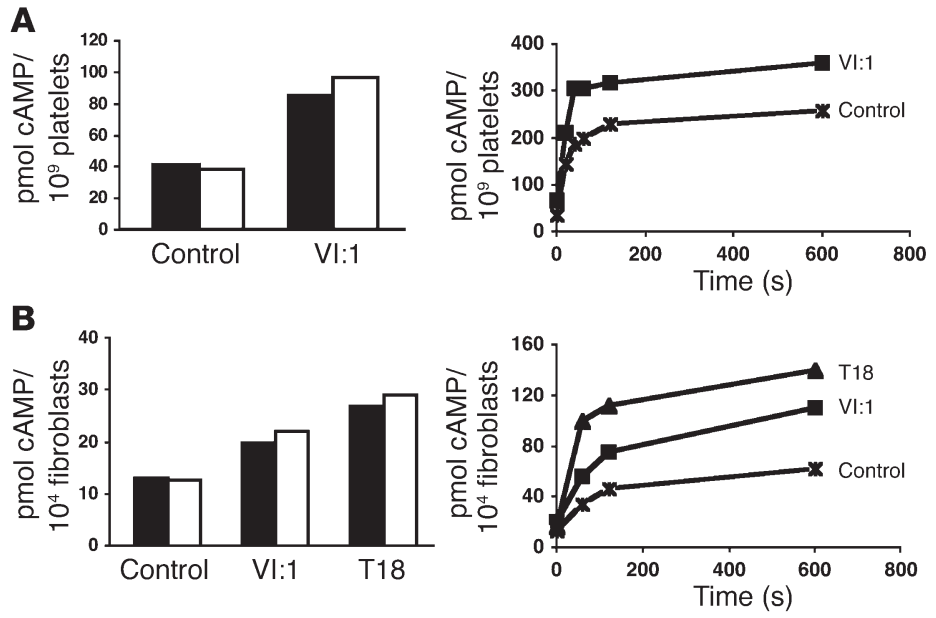

\section{Figure 2}

Adenylyl cyclase activity. (A) Measurements of cAMP levels under basal conditions were performed in duplicate (left panel) or after stimulation with iloprost $(1 \mathrm{ng} / \mathrm{ml}$ ) for various time intervals (right panel) in platelets from VI:1 (squares) or an unrelated control (asterisks). (B) Measurements of cAMP levels under basal conditions, performed in duplicate (left panel) or after stimulation with isoproterenol $(1 \mu \mathrm{M})$ for various time intervals (right panel) in fibroblasts from VI:1 (squares), a patient with a complete trisomy 18 (T18; triangles), or an unrelated control (asterisks). All cAMP measurements were performed in the presence of the phosphodiesterase inhibitor IBMX $(100 \mu \mathrm{M})$. monosomy 20p. Patients and/or their legal representatives consented to the determination of platelet and fibroblast Gs activity and the molecular analysis.

Adenylyl cyclase activity in platelets and fibroblasts. The propositus (VI:1) has a disturbed platelet function evidenced by a gain-of-Gs activity measured in the platelet aggregation inhibition test, which is seemingly similar to what we previously described for patients with an inducible Gs hyperfunction syndrome $(13,14)$. Platelets from the patients (VI:1 and V:4) with the partial trisomy 18p and monosomy 20p had a significantly increased sensitivity toward the Gs agonist iloprost (Table 1), while platelets from the family members (IV:5 and V:3) with the balanced translocation showed a moderately increased sensitivity. The $\mathrm{IC}_{50}$ value for member IV: 6 with the normal karyotype is within the range of the $\mathrm{IC}_{50}$ values from 22 unrelated controls.

An important difference between patients from this family and the patients with the inducible Gs hyperfunction we reported previously is their decreased sensitivity toward different platelet agonists (collagen, ADP, and U46619). The collagen aggregation was further studied in more detail in all family members and different unrelated controls. The collagen concentration needed to obtain $50 \%$ aggregation of platelets from $\mathrm{V}: 4$ and VI: 1 is significantly higher than for platelets from controls or the normal member IV:6 (Table 1). The reactivity of platelets from IV:5 and V:3 toward collagen is again mildly affected. This suggested that in the platelets of the propositus (VI:1), adenylyl cyclase is already activated under basal conditions. In addition to an increased cAMP response to iloprost, patient VI: 1 indeed showed higher basal cAMP levels (Figure 2A). We also performed cAMP measurements in fibroblasts from VI: 1 and found a similarly increased basal and stimulated cAMP response (Figure 2B). Interestingly, measurement of the adenylyl cyclase activity in fibroblasts from an unrelated patient with a complete trisomy 18 showed similarly increased basal and stimulated cAMP levels (Figure 2B).

$P A C A P(1-38) m R N A$ and protein overexpression. A genetic defect in one of the Gs $\alpha$ isoforms could be excluded $(13,24,25)$. Patient VI:1 has a normal Gs $\alpha$ and XL $\alpha$ s protein expression level, and the coding sequence for the Gs $\alpha$ gene and XL-exon 1 are complete-
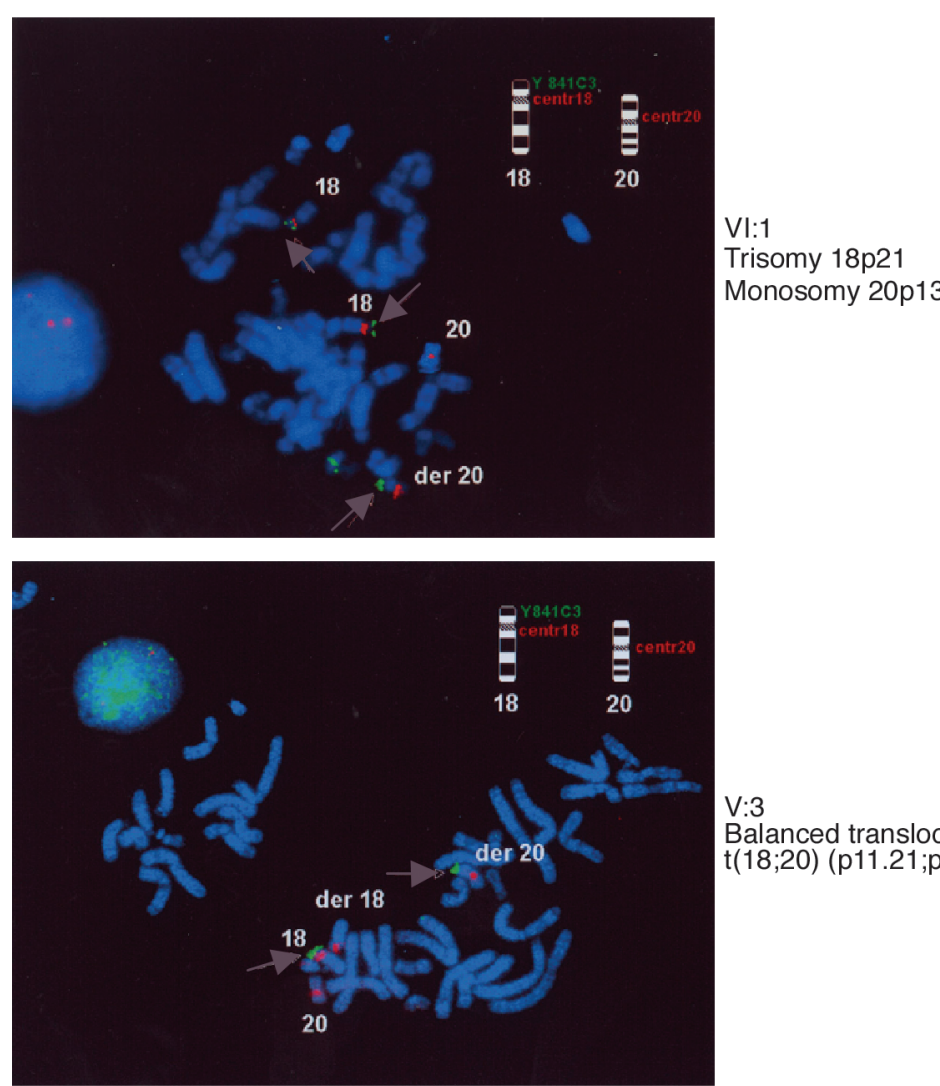

$\mathrm{V}: 3$

Balanced translocation $t(18 ; 20)(p 11.21 ; p 13)$

Figure 3

Localization of the PACAP gene by FISH. Two-color FISH with probe YAC 841C3 (green) and two centromeric probes for chromosomes 18 and 20 (red) for patient VI:1 and his mother $\mathrm{V}: 3$. The arrows point to the PACAP signals. 

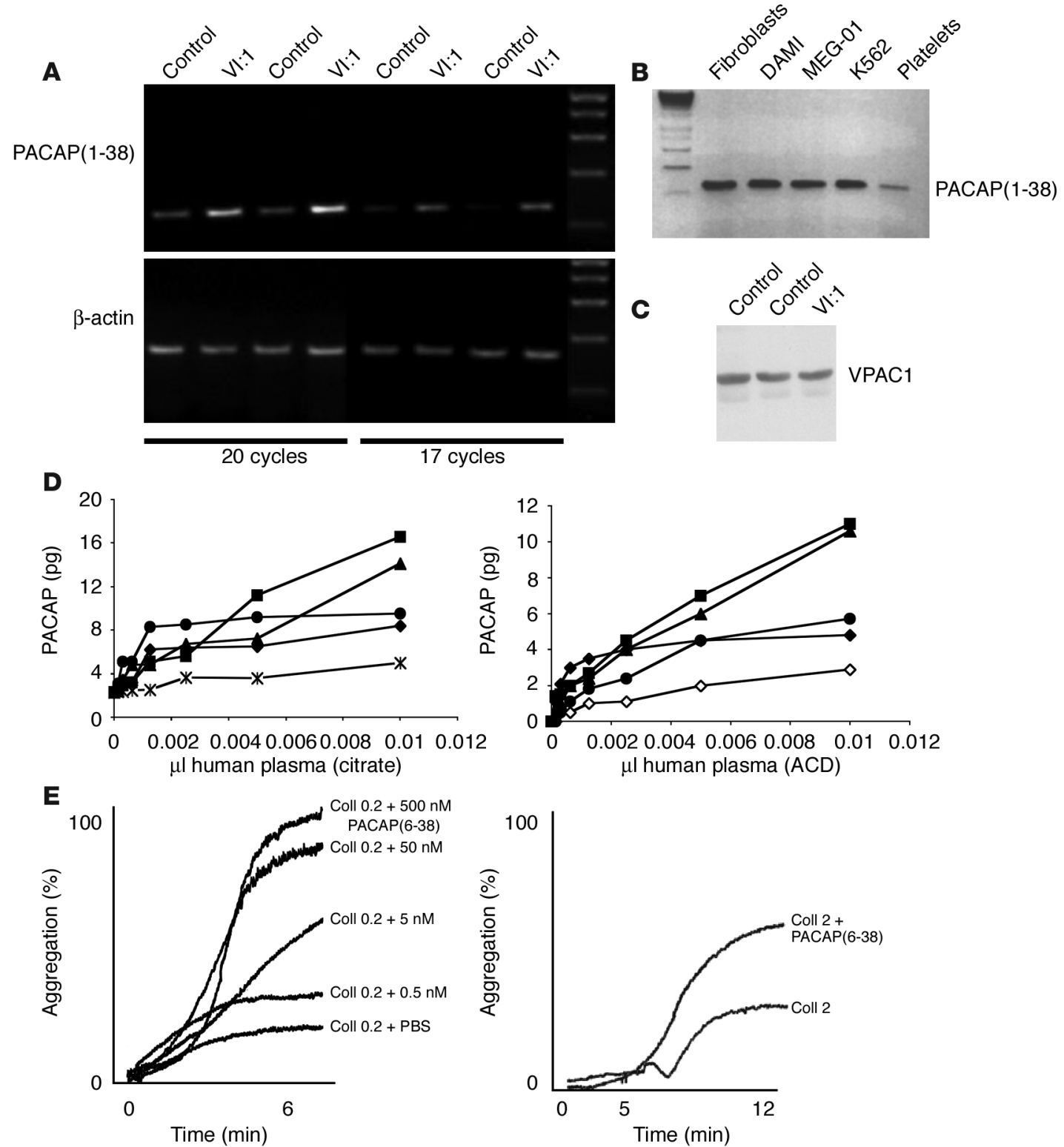

Figure 4

PACAP detection in fibroblasts and plasma. (A) Semiquantitative RT-PCR using 20 and 17 cycles showed PACAP(1-38) overexpression in fibroblasts from patient $\mathrm{Vl}: 1$ compared with the control. RT-PCR was performed on two separate fibroblast samples, and $\beta$-actin is the internal control. (B) PACAP mRNA detection in fibroblasts, megakaryocytic cell lines DAMI, MEG-01, and K562, and platelets was performed by RT-PCR. (C) Immunoblot analysis of the VPAC1 receptor $(58 \mathrm{kDa})$ in platelets from two unrelated controls and patient VI:1. (D) PACAP detections by ELISA in plasma from citrate (left panel) or ACD (right panel) blood from VI:1 (squares) and V:4 (triangles) or IV:5 (circles) and V:3 (filled diamonds) versus a citrated plasma pool (asterisks) or IV:6 (open diamonds). (E) The left panel shows the dose-dependent stimulation by PACAP(6-38) of the collagen-induced (0.2 $\mu \mathrm{g} / \mathrm{ml})$ aggregation of normal human platelets; representative tracings of five separate experiments are shown. The right panel illustrates the effect of PACAP(6-38) on collagen-induced $(2 \mu \mathrm{g} / \mathrm{ml})$ platelet aggregation in patient Vl:1. Coll, collagen.

Human skin fibroblasts express PACAP(1-38) and the PACAP type 1 receptor (26). PACAP(1-38) mRNA was overexpressed in fibroblasts from patient VI:1, as determined by semiquantitative RT-PCR (Figure 4A). PACAP is expressed in the human megakaryocytic cell lines MEG-01, DAMI, and K562, and a rather weak expression was found in control platelets by RT-PCR (Figure 4B). Western blot analysis further revealed that platelets express the VPAC1 receptor, the levels of which were normal in the patient (Figure 4C). PACAP(1-38) was detected in human plasma by ELISA; significantly higher levels were found in patients VI: 1 and V:4, and moderately increased levels in IV:5 and V:3, in comparison with a plasma pool of unrelated controls or IV:6 (Figure 4D).

Physiological role of PACAP in normal human platelets. In another set of experiments, the patient phenotype was reversed, i.e., the activity of PACAP was neutralized by addition of the PACAP antagonist PACAP(6-38). PACAP(6-38) enhanced the collageninduced aggregation of normal human platelets in a dose-dependent manner (Figure 4E) and decreased basal cAMP levels (data 


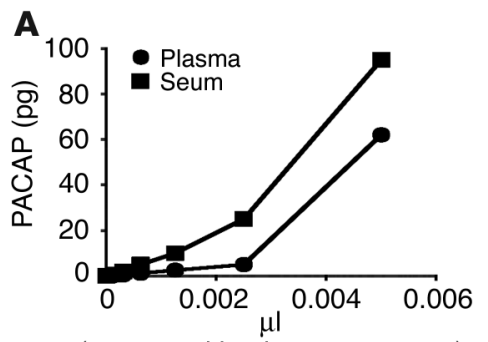

(measured in plasma or serum)

D

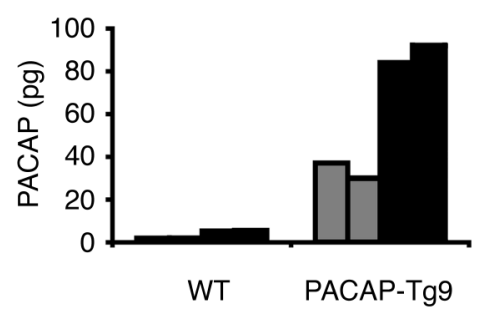

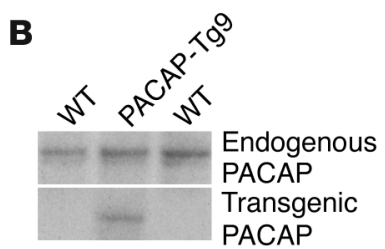

C

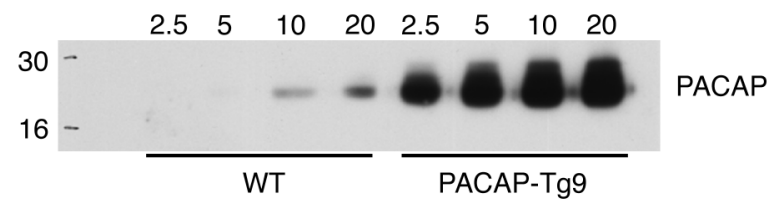

$\mathbf{E}$

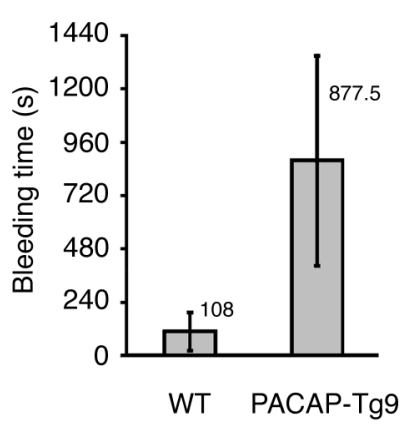

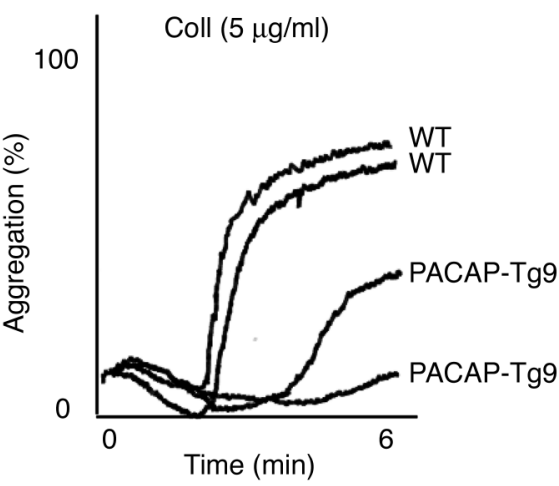

Figure 5

Increased levels of PACAP and platelet function. (A) PACAP levels detected by ELISA in plasma (circles) or serum (squares) pooled from three mice. (B) Southern blot analysis of tailsnip DNA. (C) Immunoblot analysis of the prepro-protein of PACAP $(20 \mathrm{kDa})$ in washed platelet releasates pooled from five wild-type versus five PACAP-overexpressing (PACAP-Tg9) mice. (D) PACAP levels detected by ELISA in $0.00125 \mu \mathrm{l}$ plasma (gray bars) or $0.00125 \mu \mathrm{l}$ serum (black bars) pooled from three wild-type versus three PACAP-Tg9 mice, performed in duplicate. (E) Left panel: The mean bleeding time \pm SD for either wild-type or PACAP-Tg9 mice (ten animals in each group; unpaired $t$ test; $P=0.0001$ ). Right panel: The collagen-induced $(5 \mu \mathrm{g} / \mathrm{ml})$ platelet aggregations in hirudinized PRP from two wild-type and two PACAP-Tg9 mice are shown.

not shown). The PACAP antagonist also induced a partial but consistent improvement of the collagen-induced platelet aggregation in the patient (Figure 4E).

Role for PACAP in platelet function, studied in mice. Different mouse models were studied to further evaluate the role of circulating PACAP(1-38) as a physiological inhibitor of platelet function. In mice the levels of PACAP, detected by ELISA, were higher than in human plasma; moreover, we found that PACAP levels in serum were 1.5-fold higher than in plasma, which suggested that platelets might contribute significantly to circulating PACAP(1-38) levels (Figure 5A).

To examine the effect of increased platelet PACAP levels on platelet function, transgenic mice with a PACAP overexpression in the megakaryocyte lineage were developed. The mouse PACAP gene was placed behind the platelet GPIIb promoter to drive megakaryocyte-specific expression (16). Southern blotting identified three transgenic founders with germ-line transmission of the transgene, and the founder line Tg9, with high levels of PACAP platelet expression, was selected and characterized in detail (Figure 5B). Both platelet and circulating PACAP levels were increased in the PACAP-overexpressing mice as detected by immunoblot analysis of PACAP in washed platelet releasates (Figure 5C) and ELISA detection of PACAP in serum and plasma (Figure 5D), respectively. Moreover, as for the patients, the transgenic PACAP-overexpressing mice presented an increased bleeding time and showed a decreased sensitivity toward collagen-induced aggregation (Figure 5E).

Mouse platelets incubated with anti-PACAP antibodies $(10 \mu \mathrm{g} / \mathrm{ml})$ showed an enhanced response to collagen (data not shown), and mice subcutaneously injected with anti-PACAP or anti-VPAC1 antibodies showed an enhanced response to ADP or collagen 7 days after the last injection (Figure 6, A and B). Platelets isolated from PACAPdeficient mice consistently had mildly (not significantly) decreased basal cAMP levels (wild-type mice, $133.2 \pm 15.2$ pmol cAMP per $10^{9}$ platelets; PACAP-deficient mice, $103.4 \pm 6.39$ pmol cAMP per $10^{9}$ platelets) and also showed an increased sensitivity toward collagen stimulation during aggregation studies compared with platelets from control mice (Figure 6C).

\section{Discussion}

We report the hematological consequences of PACAP overexpression, as a consequence of a partial trisomy 18p. This neuropeptide and its receptors are widely distributed, and therefore PACAP exerts pleiotropic physiological functions. Indeed, PACAP has been implicated in a wide range of biological processes, including reproductive, development, growth, cardiovascular, respiratory, and digestive functions, immune responses, and circadian rhythms (2). Our two patients with increased PACAP plasma levels, an increased bleeding tendency, and a mild thrombocytopenia also suffer from several neurological, endocrinological, and gastrointestinal defects. Further studies are needed to define whether these three latter defects are a consequence of the PACAP overexpression and reflect the pathophysiological activity of this peptide or are the result of other genes involved in the unbalanced translocation. However, we show in these patients, and also in both PACAP knockout and PACAP-overexpressing mice, that circulating PACAP modulates normal platelet function, and we 


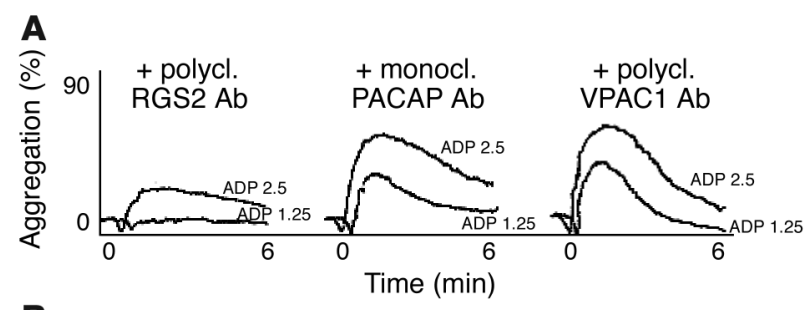

B
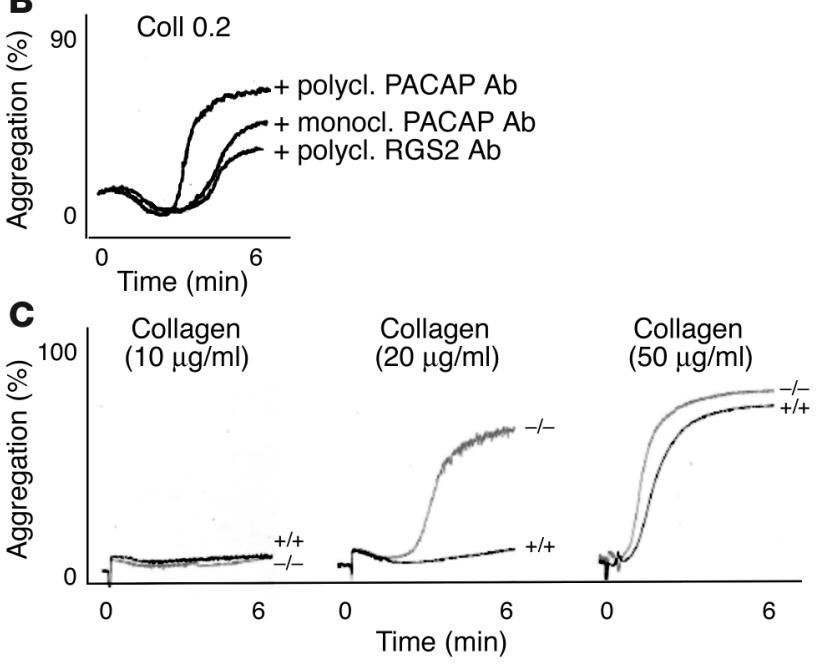

Figure 6

Decreased levels of PACAP and platelet function. (A) Platelet aggregation induced with a low concentration of ADP $(1.25$ and $2.5 \mu \mathrm{M})$ using PRP from mice injected with the indicated antibodies. (B) Platelet aggregation induced with a low concentration of collagen $(0.2 \mu \mathrm{g} / \mathrm{ml})$ using PRP from mice injected with the indicated antibodies. Platelet aggregation was performed in hirudinized PRP pooled from five mice of each group, normalized to $250 \times 10^{9}$ platelets per liter. (C) Platelet aggregation induced with the indicated concentrations of collagen using citrated PRP from either normal (+/+) or PACAP-deficient (-/-) mice. Platelet aggregation was performed in PRP pooled from three mice in each group, normalized to $250 \times 10^{9}$ platelets per liter. These tracings were representative of two independent experiments. The difference in base-line response to collagen between Figure $5 \mathrm{E}$ and Figure $6 \mathrm{C}$ could be related to the different anticoagulants used or the mouse strains. polycl., polyclonal; monocl., monoclonal.

have identified this Gs agonist as a new physiological inhibitor of platelet aggregation.

Many pharmacological responses to PACAP infusion and its effect on the CNS, the pituitary gland, and the gastrointestinal system have already been studied in detail. PACAP is thought to act within the hypothalamus as a neurotransmitter or neuromodulator to regulate the secretion of neurohypophysial and hypophysiotropic neurohormones. Intracerebroventricular injection of PACAP results in a dose-dependent elevation of plasma vasopressin and release of oxytocin (27); injection of PACAP in the medial basal hypothalamus suppresses luteinizing hormone release (28), while central administration of PACAP produces an increase in somatostatin, gonadotropin-releasing hormone, and corticotropin-releasing factor (29). PACAP can stimulate cAMP formation in pituitary cells and releases growth hormone, folliclestimulating hormone, and adrenocorticotropic hormone $(30,31)$. The effects of PACAP on the gastrointestinal system relate to the secretory activity of exocrine and endocrine cells; in addition,
PACAP induces a relaxation of gastric smooth muscles, causing a decrease of gastric motility $(32,33)$. PACAP also plays a role in the cardiovascular system by regulating blood pressure (34), but hematological changes upon PACAP administration to animals have not been documented.

PACAP-deficient mice manifest an altered psychomotor behavior, defects in female reproduction, a dysfunction of lipid and carbohydrate metabolism, and early death (20, 35-37). Knockout models for the PACAP receptors PAC1 and VPAC2 show altered emotional behavior, decreased female fertility and/or growth retardation, an increased basal metabolic rate, a role of PACAP in the relaxation in the proximal colon, and a disturbed circadian function (38-43). None of these studies reports on a potential role for PACAP in hematology. Platelets, however, only express the PACAP receptor VPAC1, for which a knockout model does not yet exist. In this study we show, for the first time to our knowledge, that platelets from PACAP-deficient mice have an increased sensitivity toward collagen stimulation. In contrast, platelets from mice with megakaryocyte-specific overexpression of PACAP have a decreased sensitivity toward collagen. PACAP mRNA is detected in platelets, and, in the mouse, serum PACAP levels are higher than in plasma. Furthermore, PACAP could be detected by immunoblotting in washed platelet releasates, suggesting that PACAP may be released from platelets following activation. This could then constitute an autocrine pathway, which would dampen the extent of activation following platelet stimulation.

In the present family, we observed an unbalanced segregation of the reciprocal translocation $\mathrm{t}(18 ; 20)$ (p21;p13) with a variable clinical phenotype. The propositus, VI:1, and his uncle, $\mathrm{V}: 4$, have a partial trisomy $18 \mathrm{p}$ and monosomy $20 \mathrm{p}$ associated with the fullblown clinical picture of severe mental retardation and a severe bleeding tendency, while the propositus's mother, V:3, and maternal grandfather, IV:5, who have a balanced translocation, have borderline IQs with no clinical bleeding problems despite a moderately abnormal platelet function. In accordance with the clinical observations, patients VI: 1 and V:4 have very high PACAP plasma levels, increased basal cAMP levels, and a platelet Gs hyperfunction, while these parameters are only mildly disturbed in family members V:3 and IV:5. It is difficult to presume what causes these increased PACAP expression levels. If an additional copy of the PACAP gene on chromosome 18p in patients VI: 1 and $\mathrm{V}: 4$ results in PACAP overexpression, it is hard to understand why family members with the balanced translocation have moderately raised PACAP and a disturbed platelet function. Further studies are needed to evaluate the role of potential regulatory elements located in the translocation region of chromosome $18 \mathrm{p}$ or to exclude the possibility that family members V:3 and IV:5 have an unusual chromosomal mosaicism that would account for their intermediate phenotype. Such a hidden mosaicism for a supernumerary derivative chromosome in a carrier of a familial reciprocal translocation was previously described in a patient with mental retardation and congenital malformations (44). The fact that PACAP expression in the trisomy 18 fibroblasts was elevated supports a PACAP gene-dosage effect.

By this genetic study in patients with a pronounced bleeding tendency, we identified PACAP as a new physiological inhibitor of platelet activation, a conclusion experimentally validated via the use of different mouse models. This knowledge may lead to new modalities in the prevention and treatment of thrombosis and bleeding, based on the administration of PACAP or its antagonists. 


\section{Acknowledgments}

This work was supported by research grants G.0371.01 and KAN20001523100 from the Fonds voor Wetenschappelijk Onderzoek-Vlaanderen (FWO-Vlaanderen) and OT/00/25 and GOA/2004/09 from Katholieke Universiteit Leuven. K. Freson is a postdoctoral fellow of the FWO, and C. Van Geet holds a clinical-fundamental research mandate from the FWO. We gratefully acknowledge Hisashi Kato for his valuable advice and support. This research was supported, in part, by a Grant-in-Aid for Scientific Research and Exploratory Research from the Ministry of Education, Culture, Sports, Science and Technology of
Japan to A. Baba and H. Hashimoto, and by grants from AstraZeneca Pharmaceuticals LP and the Naito Foundation to H. Hashimoto.

Received for publication June 20, 2003, and accepted in revised form January 13, 2004.

Address correspondence to: Kathleen Freson, Center for Molecular and Vascular Biology, University of Leuven, Herestraat 49, B-3000 Leuven, Belgium. Phone: 32-16-345775; Fax: 32-16-345990; E-mail: kathleen.freson@med.kuleuven.ac.be.
1. Miyata, A., et al. 1989. Isolation of a novel 38 residuehypothalamic polypeptide which stimulates adenylate cyclase in pituitary cells. Biochem. Biophys. Res. Commun. 164:567-574.

2. Vaudry, D., et al. 2000. Pituitary adenylate cyclaseactivating polypeptide and its receptors: from structure to functions. Pharmacol. Rev. 52:269-324.

3. Hosoya, M., et al. 1992. Structure of the human pituitary adenylate cyclase-activating polypeptide (PACAP) gene. Biochim. Biophys. Acta. 1129:199-206.

4. Buscail, L., et al. 1990. Presence of highly selective receptors for PACAP (pituitary adenylate cyclase activating peptide) in membranes from the rat pancreatic acinar cell line AR 4-2J. FEBS Lett. 262:77-81.

5. Banks, W.A., Kastin, A.J., Komaki, G., and Arimura, A. 1993. Passage of pituitary adenylate cyclase activating polypeptide1-27 and pituitary adenylate cyclase activating polypeptide1-38 across the bloodbrain barrier. J. Pharmacol. Exp. Ther. 267:690-696.

6. Tams, J.W., Johnsen, A.H., and Fahrenkrug, J. 1999. Identification of pituitary adenylate cyclase-activating polypeptide1-38-binding factor in human plasma, as ceruloplasmin. Biochem. J. 341:271-276.

7. Somogyvari-Vigh, A., Svoboda-Teet, J., Vigh, S., and Arimura, A. 1998. Is an intravenous bolus injection required prior to initiating slow intravenous infusion of PACAP38 for prevention of neuronal death induced by global ischemia? The possible presence of a binding protein for PACAP38 in blood. Ann. N. Y. Acad. Sci. 865:595-600.

8. Lutz, E.M., et al. 1993. The VIP2 receptor: molecular characterisation of a cDNA encoding a novel receptor for vasoactive intestinal peptide. FEBS Lett. 334:3-8.

9. Pisegna, J.R., and Wank, S.A. 1993. Molecular cloning and functional expression of the pituitary adenylate cyclase-activating polypeptide type I receptor. Proc. Natl. Acad. Sci. U. S. A. 90:6345-6349.

10. Hashimoto, H., Ishihara, T., Shigemoto, R., Mori, K., and Nagata, S. 1993. Molecular cloning and tissue distribution of a receptor for pituitary adenylate cyclase-activating polypeptide. Neuron. 11:333-342.

11. Spengler, D., et al. 1993. Differential signal transduction by five splice variants of the PACAP receptor. Nature. 365:170-175.

12. Kis, B., et al. 1999. Effects of pituitary adenylate cyclase-activating polypeptide on the cyclooxygenase pathway of rat platelets and on platelet aggregation. Prostaglandins Other Lipid Mediat. 58:103-112.

13. Freson, K., et al. 2001. Genetic variation of the extralarge stimulatory $G$ protein $\alpha$-subunit leads to Gs hyperfunction in platelets and is a risk factor for bleeding. Thromb. Haemost. 86:733-738.

14. Freson, K., et al. 2003. Functional polymorphisms in the paternally expressed XLalphas and its cofactor ALEX decrease their mutual interaction and enhance receptor-mediated cAMP formation. Hum. Mol. Genet. 15:1121-1130.

15. Denarier, E., Martin, F., Martineau, S., and Marguerie, G. 1993. PCR cloning and sequence of the murine GPIIb gene promoter. Biochem. Biophys. Res. Commun. 195:1360-1364.

16. Tronick-Le Roux, D., Roullot, V., Schweitzer, A., Berthier, R., and Marguerie, G. 1995. Suppression of erythro-megakaryocytopoiesis and the induction of reversible thrombocytopenia in mice transgenic for the thymidine kinase gene targeted by the platelet glycoprotein IIb promoter. J. Exp. Med. 181:2141-2151.

17. Oury, C., et al. 2003. Overexpression of the platelet $\mathrm{P} 2 \mathrm{X} 1$ ion channel in transgenic mice generates a novel prothrombotic phenotype. Blood. 101:3969-3976.

18. Holvoet, P., Danloy, S., and Collen, D. 1997. Role of the carboxy-terminal domain of human apolipoprotein AI in high-density-lipoprotein metabolism: a study based on deletion and substitution variants in transgenic mice. Eur. J. Biochem. 245:642-647.

19. Foster, C.J., et al. 2001. Molecular identification and characterization of the platelet ADP receptor targeted by thienopyridine antithrombotic drugs. J. Clin. Invest. 107:1591-1598.

20. Hashimoto, H., et al. 2001. Altered psychomotor behaviors in mice lacking pituitary adenylate cyclaseactivating polypeptide (PACAP). Proc. Natl. Acad. Sci. U. S. A. 98:13355-13360

21. Baba, A., Saga, H., and Hashimoto, H. 1993. Inhibitory glutamate response on cyclic AMP formation in cultured astrocytes. Neurosci. Lett. 149:182-184.

22. Pinkel, D., Straume, T., and Gray, J.W. 1986. Cytogenetic analysis using quantitative, high-sensitivity, fluorescence hybridization. Proc. Natl. Acad. Sci. U. S. A. 83:2934-2938.

23. Chang, E., Welch, S., Luna, J., Giacalone, J., and Francke, U. 1993. Generation of a human chromosome 18-specific YAC clone collection and mapping of 55 unique YACs by FISH and fingerprinting. Genomics. 17:393-402.

24. Hayward, B.E., Moran, V., Strain, L., and Bonthron, D.T. 1998. Bidirectional imprinting of a single gene: GNAS1 encodes maternally, paternally, and biallelically derived proteins. Proc. Natl. Acad. Sci. U. S. A 95:15475-15480.

25. Kehlenbach, R.H., Matthey, J., and Huttner, W.B. 1994. XLos is a new type of G protein. Nature 372:804-809.

26. Steinhoff, M., et al. 1999. Identification of pituitary adenylate cyclase activating polypeptide (PACAP) and PACAP type 1 receptor in human skin: expression of PACAP-38 is increased in patients with psoriasis. Regul. Pept. 80:49-55.

27. Lutz-Bucher, B., Monnier, D., and Koch, B. 1996. Evidence for the presence of receptors for pituitary adenylate cyclase-activating polypeptide in the neurohypophysis that are positively coupled to cyclic AMP formation and neurohypophyseal hormone secretion. Neuroendocrinology. 64:153-161.

28. Anderson, S.T., Sawangjaroen, K., and Curlewis, J.D 1996. Pituitary adenylate cyclase-activating polypeptide acts within the medial basal hypothalamus to inhibit prolactin and luteinizing hormone secretion. Endocrinology. 137:3424-3429.

29. Li, P., Grinevich, V., Fournier, A., and Pelletier, G. 1996. Effects of pituitary adenylate cyclase-activating polypeptide (PACAP) on gonadotropin-releasing hormone and somatostatin gene expression in the rat brain. Mol. Brain Res. 41:157-162.

30. Goth, M.I., Lyons, C.E., Canny, B.J., and Thorner,
M.O. 1992. Pituitary adenylate cyclase-activating polypeptide, growth hormone (GH)-releasing peptide and GH-releasing hormone stimulate GH release through distinct pituitary receptors. Endocrinology. 130:939-944.

31. Hart, G.R., Gowing, H., and Burrin, J.W. 1992. Effects of a novel hypothalamic peptide, pituitary adenylate cyclase-activating polypeptide, on pituitary hormone release in rats. J. Endocrinol. 134:33-41.

32. Katsoulis, S., et al. 1996. Inhibitory transmission in guinea pig stomach mediated by distinct receptors for pituitary adenylate cyclase-activating polypeptide. J. Pharmacol. Exp. Ther. 278:199-204.

33. Mizuta, K., Ozawa, M., Aono, M., and Moriga, M. 1994. Centrally administered PACAP stimulated gastric acid secretion in anesthetized rats. Biomed. Res. 15:253-256.

34. Nandha, K.A., Benito-Orfila, M.A., Smith, D.M., Ghaei, M.A., and Bloom, S.R. 1991. Action of pituitary adenylate cyclase-activating polypeptide and vasoactive intestinal polypeptide on the rat vascular system: effects on blood pressure and receptor binding. J. Endocrinol. 129:69-73.

35. Shintani, N., et al. 2002. Defects in reproductive functions in PACAP-deficient female mice. Regul. Pept. 109:45-48.

36. Gray, S.L., Cummings, K.J., Jirik, F.R., and Sherwood, N.M. 2001. Targeted disruption of the pituitary adenylate cyclase-activating polypeptide gene results in early postnatal death associated with dysfunction of lipid and carbohydrate metabolism. Mol. Endocrinol. 15:1739-1747.

37. Hamelink, C., et al. 2002. Pituitary adenylate cyclaseactivating polypeptide is a sympathoadrenal neurotransmitter involved in catecholamine regulation and glucohomeostasis. Proc. Natl. Acad. Sci. U. S. A 99:461-466.

38. Otto, C., et al. 2001. Altered emotional behavior in PACAP-type-I-receptor-deficient mice. Brain Res. Mol. Brain Res. 92:78-84.

39. Jamen, F., et al. 2000. PAC1 null females display decreased fertility. Ann. N. Y. Acad. Sci. 921:400-404.

40. Hannibal, J., et al. 2001. Dissociation between lightinduced phase shift of the circadian rhythm and clock gene expression in mice lacking the pituitary adenylate cyclase activating polypeptide type 1 receptor. J. Neurosci. 21:4883-4890.

41. Mukai, K., et al. 2002. PAC1 receptor-mediated relaxation of longitudinal muscle of the mouse proximal colon. Jpn. J. Pharmacol. 90:97-100.

42. Asnicar, M.A., et al. 2002. Vasoactive intestinal polypeptide/pituitary adenylate cyclase-activating peptide receptor 2 deficiency in mice results in growth retardation and increased basal metabolic rate. Endocrinology. 143:3994-4006

43. Harmar, A.J., et al. 2002. The VPAC(2) receptor is essential for circadian function in the mouse suprachiasmatic nuclei. Cell. 109:497-508.

44. Dufke, A., Mayrhofer, H., Enders, H., Kaiser, P., and Leipoldt, M. 2001. Unusual chromosomal mosaicism as a cause of mental retardation and congenital malformations in a familial reciprocal translocation carrier, $\mathrm{t}(17 ; 22)(\mathrm{q} 24.2 ; \mathrm{q} 11.23)$. Cytogenet. Cell Genet. 93:168-170. 